\section{Ambiente alimentar: validação de método de mensuração e caracterização em território com o Programa Academia da Saúde}

\author{
Food environment: validation of a method for \\ measurement and characterization in the \\ territory with the Health Academy Program
}

\author{
Ambiente alimentario: validación del método de \\ medición y caracterización en territorios con el \\ Programa Academia de la Salud
}

Bruna Vieira de Lima Costa 1

Patrícia Pinheiro de Freitas 1

Mariana Carvalho de Menezes

Larissa Morelli Ferraz Guimarães 1

Luana de Fátima Ferreira 1

Mariana dos Santos Costa Alves 1

Aline Cristine Souza Lopes 1

\section{Resumo}

O objetivo foi verificar a validade de dados secundários na investigação do ambiente alimentar e analisar as características do ambiente da comunidade e do consumidor em territórios de serviço de promoção da saúde. Estudo ecológico desenvolvido em 18 unidades do Programa Academia da Saúde de Belo Horizonte, Minas Gerais, Brasil, selecionadas por amostragem de conglomerado simples. A validação dos estabelecimentos que comercializam frutas e hortaliças, obtidos valendo-se de bases de dados públicas, foi realizada por contato telefônico, uso da ferramenta Google Street View e auditoria. As variáveis do ambiente alimentar da comunidade investigadas foram: tipo de estabelecimento e localização; e do ambiente do consumidor: disponibilidade, variedade, preço e propaganda de frutas e hortaliças, e disponibilidade e variedade de alimentos ultraprocessados; e aspectos higiênico-sanitários. Para mensurar o acesso a alimentos saudáveis, utilizou-se o índice de acesso a estes alimentos. A auditoria revelou concordância fraca (45,7\%) das bases secundárias de dados. Dos 298 estabelecimentos auditados, a maioria era sacolões e feiras-livres (61,3\%), que apresentavam maior disponibilidade de alimentos saudáveis, mas também comercializavam de forma expressiva alimentos ultraprocessados (60,7\%). Quanto às condições sanitárias, 1/3 dos estabelecimentos foi reprovado. Foi baixa a validade das bases secundárias, reforçando a necessidade de realizar auditoria nos estabelecimentos. Ademais, os estabelecimentos investigados apresentaram presença marcante de alimentos ultraprocessados e inadequadas condições higiênico-sanitárias.

Comércio; Alimentos; Saúde Urbana; Estudos de Validação

\section{Correspondência}

B. V. L. Costa

Universidade Federal de Minas Gerais.

Av. Alfredo Balena 190, Belo Horizonte, MG 30130-100, Brasil. brunavlcosta@gmail.com

1 Universidade Federal de Minas Gerais, Belo Horizonte, Brasil. 


\section{Introdução}

O ambiente alimentar é definido pelos ambientes físico (disponibilidade, qualidade e promoção), econômico (custos), político (políticas governamentais) e sociocultural (normas e comportamento), em que se vive, estuda e/ou trabalha, e que propiciam oportunidades e condições que afetam a salubridade da alimentação e o estado nutricional dos indivíduos e da comunidade 1,2 . Ele pode ser investigado no âmbito da comunidade, na qual se observa a distribuição dos estabelecimentos comerciais em número, tipo, localização e acessibilidade; e no âmbito do consumidor, que abrange o que se encontra dentro e ao redor dos estabelecimentos (qualidade, preço, propaganda, disponibilidade e variedade dos alimentos ofertados) 1 .

Estudos epidemiológicos que analisam o ambiente alimentar da comunidade geralmente utilizam dados secundários, disponíveis em cooperativas comerciais, listas telefônicas e de internet, ou bases de dados governamentais públicas. Entretanto, há questionamentos sobre a validade dessas informações e a qualidade dos resultados que geram 3.

Trabalhos realizados em países como Estados Unidos, Canadá, Dinamarca, Escócia e Grã-Bretanha apresentaram valores discrepantes de validade dos dados secundários. Os coeficientes de correlação variaram de 0,23 a 0,94, conforme a origem da informação e o local do estudo. Características como pior renda e condições socioeconômicas do território, além da qualidade das listas utilizadas, foram consideradas os principais causadores de divergências ${ }^{3}$.

Dados secundários também restringem a análise do ambiente alimentar no nível da comunidade, mas a literatura aponta que é importante ir além e analisar as características dos alimentos que os consumidores encontram nos estabelecimentos comerciais, o que somente é possível por processos de auditoria 4,5. Investigar o ambiente alimentar do consumidor permite verificar a disponibilidade e a qualidade dos alimentos nos estabelecimentos, evitando o reducionismo de rotular estabelecimentos como "saudáveis" ou "não saudáveis", considerando apenas o seu tipo, sem necessariamente conhecer os produtos ofertados 4,6.

Investigar o ambiente alimentar tanto da comunidade quanto do consumidor é fundamental na atualidade, ao considerar a limitação das análises centradas no indivíduo para estudar algo tão complexo como a alimentação e a saúde. Com base em informações sobre o território em que os indivíduos estudam/vivem/trabalham é possível delinear melhor as políticas públicas e estratégias de saúde, incluindo a criação de ambientes saudáveis. Tais aspectos reforçam a importância de se investigar o ambiente alimentar no contexto das políticas públicas, de forma a alinhar as estratégias e as ações de saúde e de alimentação, sendo a investigação dos territórios com serviços de saúde estratégicosneste sentido.

Espera-se que este artigo contribua para a literatura vigente sobre o tema ao utilizar informações validadas para a investigação conjunta do ambiente da comunidade e do consumidor, contribuindo para a compreensão e o monitoramento do ambiente alimentar, sobretudo nos territórios com serviços públicos de promoção da saúde, bem como para a formulação de políticas públicas. Para isso, ele foi delineado com os seguintes objetivos: verificar a validade de dados secundários na investigação do ambiente alimentar e analisar as características do ambiente alimentar da comunidade e do consumidor em territórios com serviço de promoção da saúde.

\section{Métodos}

Este é um estudo derivado de pesquisa intitulada Consumo de Frutas e Hortaliças em Serviços de Promoção da Saúde de Belo Horizonte, Minas Gerais: Fatores Associados e Intervenções Nutricionais, que objetivou desenvolver e avaliar as intervenções nutricionais direcionadas ao incentivo do consumo de frutas e hortaliças no Município de Belo Horizonte 7.

Belo Horizonte é dividida em nove Regiões Administrativas, nas quais são planejadas e gerenciadas todas as políticas e ações de saúde. O município tem investido em ações de saúde territorializadas, sendo a construção do sistema de informações geográficas 8 e a criação do serviço de promoção da saúde, denominados Programa Academia da Saúde (PAS), algumas de suas estratégias.

O PAS foi escolhido para a investigação do ambiente alimentar de seu território por ser um serviço de promoção da saúde da atenção primária do município, que objetiva ofertar modos saudáveis 
de vida, incluindo ações de promoção da alimentação saudável, dentre outras. Adicionalmente, esse serviço preconiza a criação de ambientes favoráveis à saúde, a atuação no território, a participação popular e a autonomia como alternativas para o enfrentamento dos determinantes sociais 9,10 .

\section{Tipo, amostra e território de estudo}

Conduziu-se um estudo ecológico em amostra representativa de unidades do PAS.

Para a seleção das unidades participantes foram utilizados os seguintes critérios de elegibilidade: funcionar no turno matutino e estar localizada em área vulnerável por serem as características predominantes do PAS no município, e não ter participado de estudos de intervenção nutricional nos últimos dois anos. Foram consideradas elegíveis 42 unidades entre as $50 \mathrm{em}$ funcionamento, sendo sorteadas, por amostra de conglomerado simples, 18 unidades, estratificadas pelas nove Regiões Administrativas do município. Essa amostra foi representativa de seu todo com $95 \%$ de confiança e erro inferior a 1,4\%, com base na estimativa de proporção populacional 7.

Para a definição dos territórios de investigação do ambiente alimentar foram criados buffers com raio de $1.600 \mathrm{~m}$ ( $1 \mathrm{milha}$ ) ao redor de cada unidade do PAS. Optou-se por utilizar $1.600 \mathrm{~m}$ por ser considerada uma distância caminhável 11 e ser bastante utilizada em estudos sobre o ambiente alimentar 12,13. Os buffers foram identificados no software ArcView (Environmental Systems Research Institute Inc., http://www.esri.com/software/arcview/), utilizando-se a posição geográfica do serviço ${ }^{7}$.

Nesses buffers foram investigados todos os estabelecimentos que comercializavam frutas e hortaliças para consumo no domicílio. A listagem de estabelecimentos foi fornecida pela Secretaria Municipal Adjunta de Arrecadação, em duas bases de dados georreferenciadas, conforme a Classificação Nacional de Atividades Econômicas (CNAE). A primeira base era relativa ao comércio atacadista e varejista de hortifrutigranjeiros, e a segunda, a mercadorias em geral, com predominância de produtos alimentícios (hipermercados, supermercados e minimercados, mercearias e armazéns). Somados à investigação dessas bases de dados públicas também foram investigados todos os estabelecimentos comerciais identificados pela equipe de pesquisa durante o estudo de campo, mas não registrados pela Prefeitura 7.

Para a identificação das feiras-livres, utilizou-se o site da Prefeitura Municipal (https://prefeitura. pbh.gov.br/).

\section{Coleta de dados}

Para avaliar o ambiente alimentar da comunidade, inicialmente procedeu-se à validação dos dados disponibilizados pelas bases públicas. Para isso, utilizou-se contato telefônico, uso da ferramenta Google Street View (https://www.google.com.br/intl/pt/streetview/) e visita in loco para confirmar a existência do estabelecimento e a comercialização de frutas e hortaliças.

$\mathrm{Na}$ investigação do ambiente alimentar da comunidade verificou-se o tipo de estabelecimento comercial, que foi classificado em: supermercados de grande cadeia, sacolões e feiras-livres, mercados locais, lojas de conveniência e padaria 14.

Para a investigação do ambiente alimentar do consumidor foi realizada auditoria nos estabelecimentos comerciais, a fim de investigar as marcadoras da alimentação saudável (frutas e hortaliças) e não saudável (alimentos ultraprocessados). Tal opção metodológica deriva da hipótese de que a maior disponibilidade, variedade, qualidade e publicidade de alimentos saudáveis, como frutas e hortaliças, podem estar associadas a um maior acesso e, consequentemente, consumo destes alimentos; enquanto que os mesmos parâmetros para produtos ultraprocessados pode estar associado ao menor consumo de alimentos saudáveis 15 .

Foram investigadas as dez frutas e hortaliças mais consumidas no município (banana, laranja, mamão, melancia, maçã, manga, abacaxi, tangerina, uva e melão) e os cinco alimentos ultraprocessados mais consumidos no Brasil (refrigerante, suco e néctar adoçado, suco em pó, biscoito recheado de chocolate e salgadinho industrializado de milho) 16 .

Para a auditoria utilizou-se instrumentos validados propostos pelo Estudo do Ambiente Obesogênico de São Paulo (ESAO-S), referentes à avaliação de estabelecimentos de comercialização de alimentos para consumo no domicílio e feiras-livres 15 . As variáveis investigadas foram: localização 
da seção, disponibilidade, variedade, preço e propaganda de frutas e hortaliças e disponibilidade, e variedade e propaganda de alimentos ultraprocessados.

As variáveis investigadas no ambiente do consumidor e seus respectivos parâmetros estão sumarizados no Quadro 1. Para a avaliação da variedade de frutas e hortaliças observou-se a presença de tipos diferentes para um mesmo item (exemplo: banana prata, nanica, maçã e da terra); e para alimentos ultraprocessados contabilizou-se o número de marcas e sabores diferentes disponíveis no estabelecimento. A avaliação das propagandas dos alimentos foi registrada pela existência de estratégias de divulgação como: balcão de degustação, demonstrador ou distribuição de amostras; bandeirolas; cartazes/banners; display; e folder 15.

Outras variáveis investigadas no ambiente alimentar do consumidor incluíram o horário de funcionamento (dia de semana e final de semana) e aspectos higiênico-sanitário dos estabelecimentos, critérios importantes para acesso e decisão para a escolha do local de compra 17,18, respectivamente. A inclusão do aspecto higiênico-sanitário é oportuna por permitir a identificação e o mapeamento da necessidade de implantação e reforço de ações de vigilância sanitária, de forma a favorecer a construção de um ambiente alimentar aprazível e saudável.

Para avaliar as condições higiênico-sanitárias dos estabelecimentos utilizaram-se parâmetros definidos na Lista de Verificação das Boas Práticas de Fabricação em Estabelecimentos Produtores/Industrializadores de Alimentos, proposta pela Agência Nacional de Vigilância Sanitária (Anvisa) 19. Foram investigadas as seguintes variáveis: acúmulo de lixo e/ou sujidades; objetos em desuso ou estranhos ao ambiente; vetores e outros animais; focos de poeira e água estagnada; estado de conservação de pisos, tetos, paredes, portas, janelas; iluminação, ventilação e manejo de resíduos.

A coleta foi realizada de abril a setembro de 2013, por equipe de pesquisa constituída por nutricionistas e graduandos em Nutrição. De posse dos endereços dos estabelecimentos comerciais de frutas e hortaliças (registrados na base pública), previamente verificados por contato telefônico e uso da ferramenta Google Street View, somados aos estabelecimentos encontrados na rota da busca durante o campo de pesquisa (não registrados na base pública), duplas de entrevistadores realizaram a auditoria. Todos os entrevistadores foram previamente treinados com base no manual de campo 14, sendo a revisão deste treinamento conduzida semestralmente.

Para a coleta dos dados, todos os responsáveis pelos estabelecimentos comerciais foram adequadamente informados sobre a pesquisa e assinaram o Termo de Consentimento Livre e Esclarecido. O estudo foi aprovado pelos Comitês de Ética em Pesquisa da Universidade Federal de Minas Gerais (0537.0.0203.000-11) e da Prefeitura de Belo Horizonte (0537.0.0203.410-11A), conforme Resolução vigente.

\section{Análise dos dados}

Realizou-se análise descritiva, e a distribuição das variáveis foi verificada pelo teste Kolmogorov-Smirnov. As variáveis contínuas com distribuição normal (horário de funcionamento dos estabelecimentos) foram descritas pela média e desvio padrão, e as demais (índice de acesso a alimentos em estabelecimentos de comercialização para consumo no domicílio - HFSI - e variedade de alimentos ultraprocessados) por mediana e intervalo interquartil $\left(\mathrm{P}_{25}-\mathrm{P}_{75}\right)$.

Um mapa de pontos foi construído com o objetivo de proporcionar a visualização dos estabelecimentos comerciais contidos na listagem da Prefeitura do município (dados secundários) e aqueles observados in loco (dados primários).

Para verificar a validade dos dados secundários, relativos à existência dos estabelecimentos comerciais, analisou-se a concordância entre as bases públicas e os dados oriundos da auditoria. Para isso, utilizou-se o coeficiente kappa com a classificação proposta por Landis \& Koch 20: concordância leve ou pobre $(0-0,19)$, concordância fraca $(0,20-0,39)$, concordância moderada $(0,40-0,59)$, concordância substancial $(0,60-0,79)$ e concordância quase perfeita $(\geq 0,80)$.

Para mensurar a disponibilidade a alimentos saudáveis, foi utilizado o HFSI. Esse índice é composto por diferentes variáveis do ambiente do consumidor (Quadro 1) e varia de 1 a 16, sendo que quanto maior o seu valor melhor será a disponibilidade de alimentos saudáveis e, consequentemente, menor a disponibilidade de alimentos ultraprocessados no território 7,14. Para comparar a mediana do HFSI entre os tipos de estabelecimentos utilizou-se o teste Kruskal-Wallis. 


\section{Quadro 1}

Variáveis do ambiente do consumidor utilizadas para compor o índice de acesso a alimentos em estabelecimentos de comercialização para consumo no domicílio (HFSI).

\begin{tabular}{|c|c|}
\hline Variável & Escore e parâmetros de avaliação \\
\hline Seção de frutas e hortaliças próxima à entrada principal & $\begin{array}{c}0 \text { - não próximo } \\
1 \text { - próximo }\end{array}$ \\
\hline Diferentes tipos de frutas & $\begin{array}{c}\text { 0 - não disponível } \\
1 \text { - } 1 \text { a } 7 \text { tipos das } 10 \text { principais estão disponíveis } \\
2-8 \text { a } 10 \text { tipos das } 10 \text { principais estão disponíveis }\end{array}$ \\
\hline Variedade de frutas & $\begin{array}{c}0 \text { - nenhuma variedade disponível } \\
1 \text { - até } 14 \text { variedades estão disponíveis } \\
2 \text { - } 15 \text { ou + variedades disponíveis }\end{array}$ \\
\hline Diferentes tipos de hortaliças & $\begin{array}{l}\text { 0 - não disponível } \\
1 \text { - } 1 \text { a } 7 \text { tipos das } 10 \text { principais estão disponíveis } \\
2-8 \text { a } 10 \text { tipos das } 10 \text { principais estão disponíveis }\end{array}$ \\
\hline Variedade de hortaliças & $\begin{array}{c}0 \text { - nenhuma variedade disponível } \\
1 \text { - até } 14 \text { variedades estão disponíveis } \\
2 \text { - } 15 \text { ou + variedades disponíveis }\end{array}$ \\
\hline Propaganda de frutas e hortaliças & $\begin{array}{c}0 \text { - não disponível } \\
1 \text { - disponível }\end{array}$ \\
\hline Disponibilidade de refrigerantes & $\begin{array}{c}0 \text { - disponível } \\
1 \text { - não disponível }\end{array}$ \\
\hline Disponibilidade sucos adoçados/pó & $\begin{array}{c}0 \text { - disponível } \\
1 \text { - não disponível }\end{array}$ \\
\hline Disponibilidade biscoitos chocolate & $\begin{array}{c}0 \text { - disponível } \\
1 \text { - não disponível }\end{array}$ \\
\hline Disponibilidade salgadinhos & $\begin{array}{c}0 \text { - disponível } \\
1 \text { - não disponível }\end{array}$ \\
\hline Propaganda alimentos ultraprocessados & $\begin{array}{c}0 \text { - disponível } \\
1 \text { - não disponível }\end{array}$ \\
\hline
\end{tabular}

Fonte: adaptado de Duran et al. 14.

Para a avaliação dos aspectos higiênico-sanitários foram utilizados os parâmetros propostos pela Resolução da Diretoria Colegiada no 275 da Anvisa 19. Dessa forma, os estabelecimentos foram classificados como "aprovados" quando apresentaram entre 76\% e 100\% de adequação dos itens avaliados; "aprovados com restrições" quando tinham de 51\% a 75\% dos itens; e "reprovados" quando atingiram menos de 50\% de adequação dos itens.

Os dados foram tabulados no programa Access (Microsoft Corp., Estados Unidos), georreferenciados pelo software Arcview (versão 10.1) e analisados nos programas Arcview e IBM SPSS for Windows, version 19.0 (IBM Corp., Armonk, Estados Unidos). Os mapas foram construídos com o auxílio do software ArcView.

\section{Resultados}

\section{Ambiente alimentar da comunidade}

A Figura 1 apresenta o fluxograma de obtenção dos dados do ambiente alimentar, considerando as diferentes bases de dados públicas e os estabelecimentos identificados durante a rota de auditoria, bem como recusas e inexistência de estabelecimentos. 
Figura 1

Fluxograma de obtenção dos dados do ambiente alimentar do território com serviço de promoção da saúde. Belo Horizonte, Minas Gerais, Brasil, 2013.

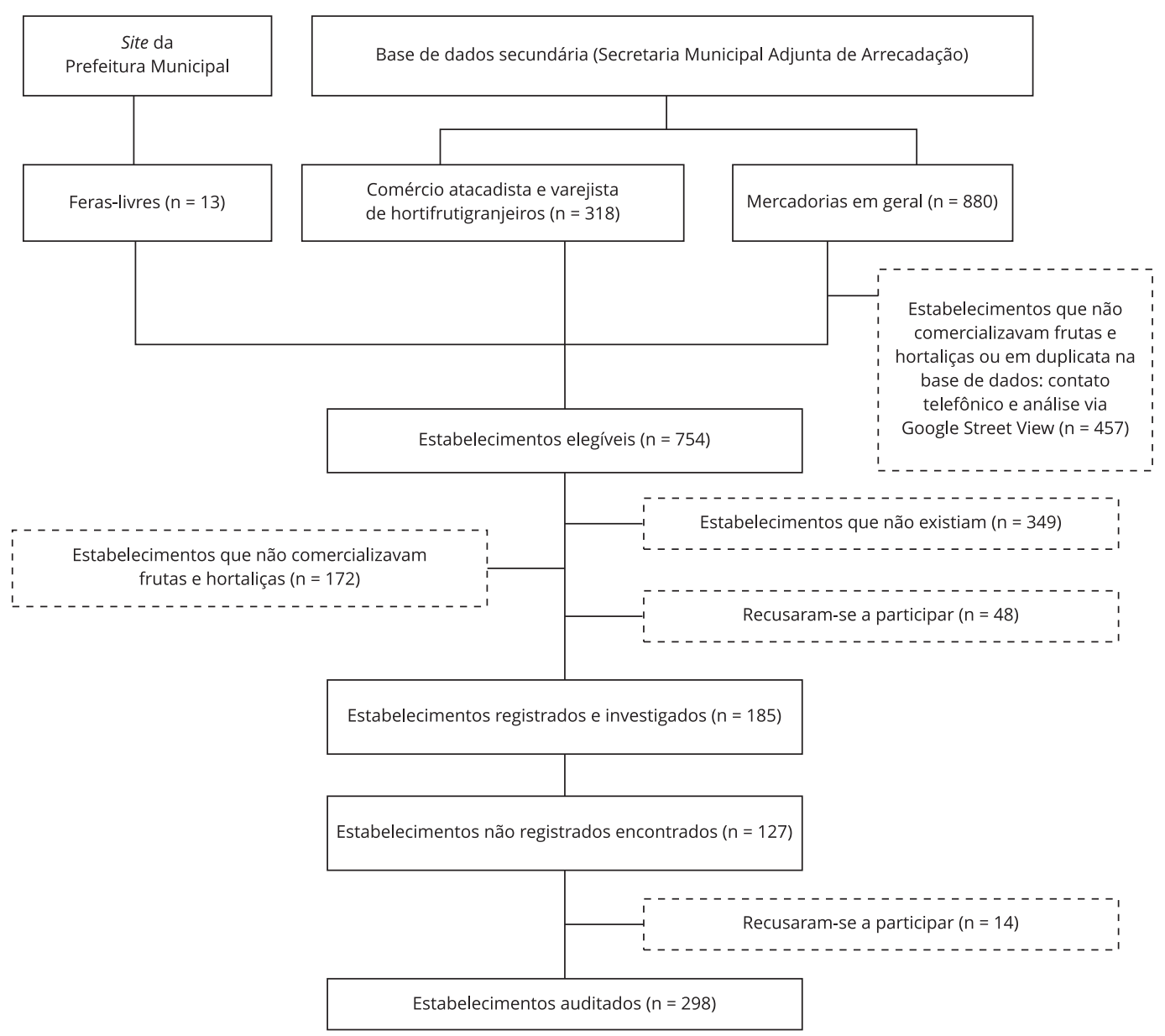

A Figura 2 mostra a distribuição espacial dos estabelecimentos comerciais disponibilizados pelo município e aqueles realmente existentes mediante verificação por auditoria. Em todos os territórios com a presença de unidades do PAS avaliados foi menor o número de estabelecimentos existentes em comparação aos indicados pelas bases de dados secundárias. A análise de validação mostrou um porcentual de concordância de apenas $45,7 \%$, sendo o coeficiente de kappa de 0,30 ( $p<0,01)$, evidenciando uma concordância fraca entre os dados das bases públicas e da auditoria.

Dos estabelecimentos auditados, a maioria era sacolões e feiras-livres (61,3\%), seguidos de mercados e supermercados de grande rede $(21,1 \%)$ e mercados locais $(17,6 \%)$.

\section{Ambiente alimentar do consumidor}

A recusa dos estabelecimentos à realização da auditoria correspondeu a 17,2\%, sendo ao final auditados 298 estabelecimentos.

Dos 298 estabelecimentos auditados (Figura 1), 62,5\% e 46,1\% apresentavam de 8 a 10 tipos das 10 principais frutas e hortaliças investigadas, respectivamente. Somente $2,1 \%$ dos estabelecimentos não disponibilizavam hortaliças e 1,2\% não comercializava frutas. Com relação à variedade, 56,5\% e 20,5\% dos locais apresentavam 15 ou mais variedades de frutas e hortaliças, respectivamente (Tabela 1). 
Figura 2

Distribuição espacial dos estabelecimentos comerciais de frutas e hortaliças disponibilizados por bases de dados secundárias versus estabelecimentos existentes verificados por auditoria no território com serviço de promoção da saúde. Belo Horizonte, Minas Gerais, Brasil, 2013.

2a) Dados secundários

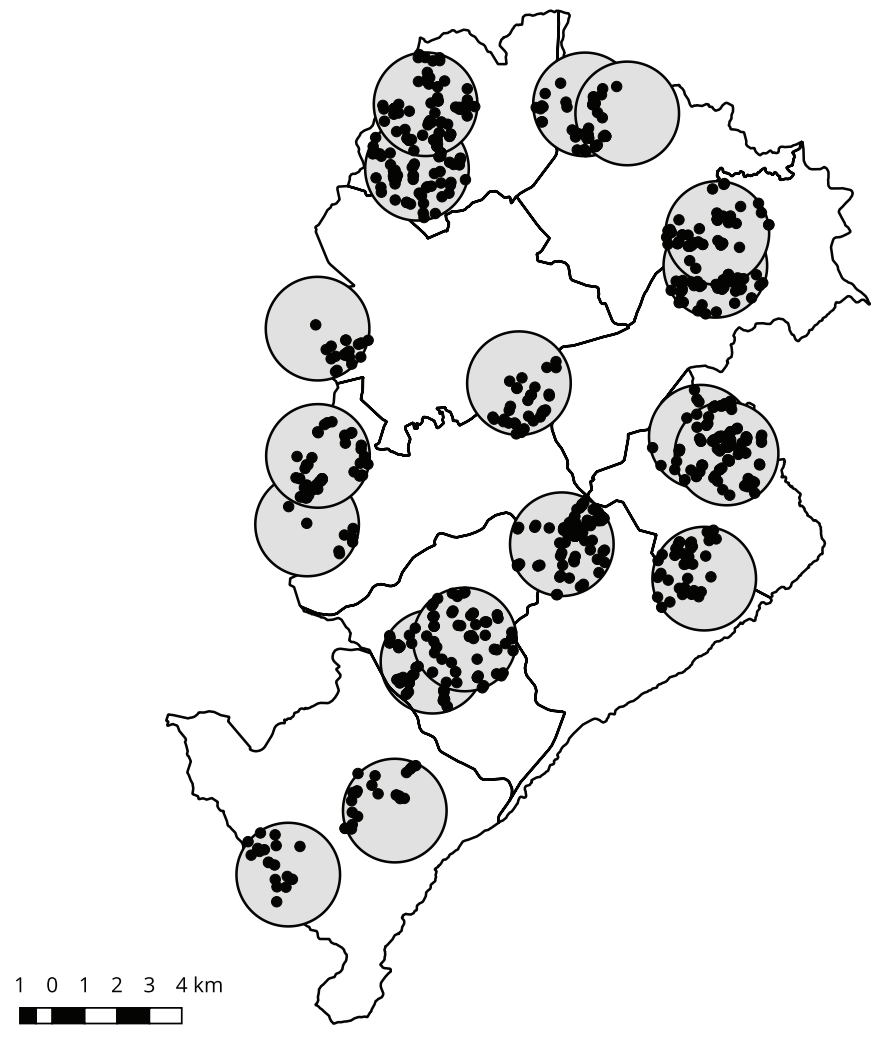

- Estabelecimentos comerciais dados secundários

Território do PAS

Regional Administrativa 2b) Estabelecimentos existentes

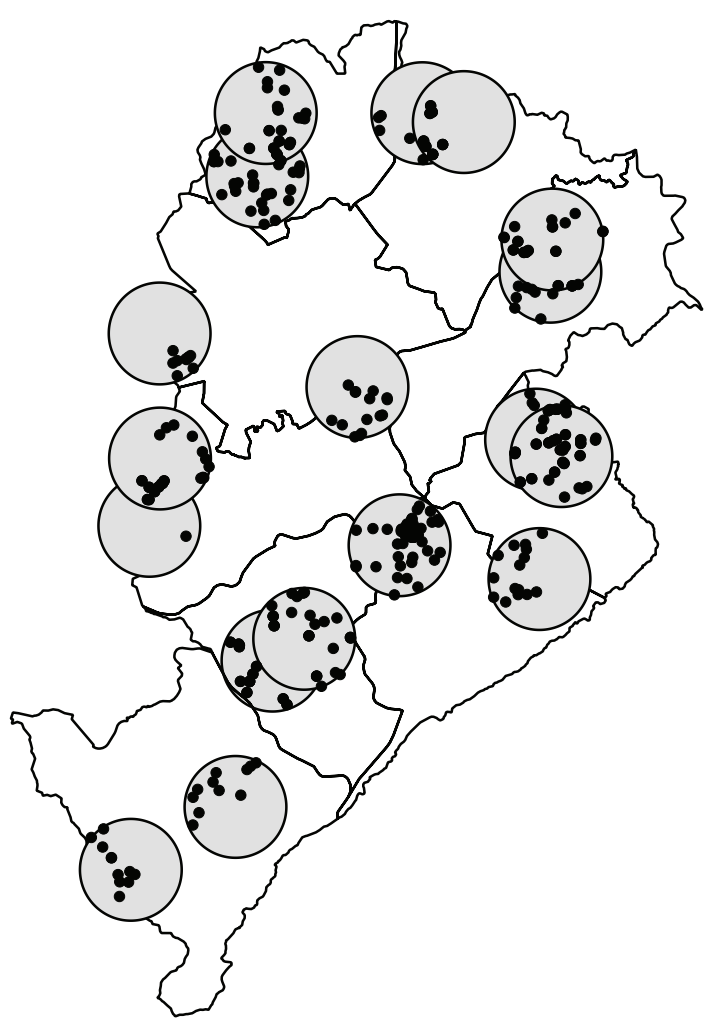

- Estabelecimentos comerciais auditados

Território do PAS

Regional Administrativa

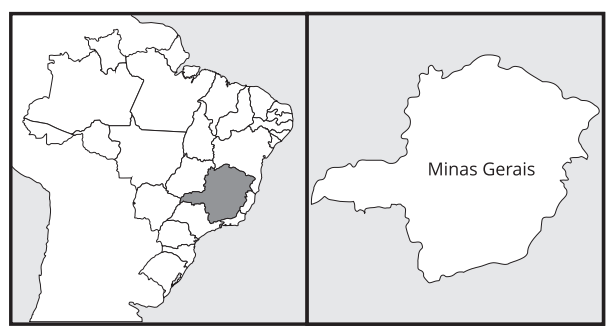

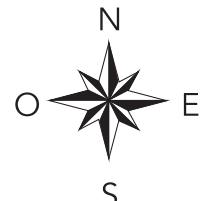

$\mathrm{S}$

PAS: Programa Academia da Saúde. 
Metade ou mais dos estabelecimentos comerciais de frutas e hortaliças (60,7\%) vendiam alimentos ultraprocessados (dados não apresentados), sendo mais comercializados os biscoitos recheados $(60,7 \%)$ e salgadinho de milho (60,7\%), seguidos de néctar/sucos adoçados e em pó $(51,5 \%)$ e refrigerantes $(49,7 \%)$. Os alimentos ultraprocessados que apresentaram maior disponibilidade de variedade foram: refrigerantes (mediana $=15 ; \mathrm{P}_{25}-\mathrm{P}_{75}$ : 9,0-24,5), suco adoçado e em pó (mediana $=7 ; \mathrm{P}_{25}-\mathrm{P}_{75}$ : $3,0-13,0)$ e biscoitos recheados (mediana $=6 ; \mathrm{P}_{25}-\mathrm{P}_{75}: 3,2-8,0$ ). Nos estabelecimentos investigados predominou a propaganda de incentivo ao consumo de alimentos ultraprocessados $(76,2 \%)$ em detrimento das frutas e hortaliças (22\%) (Tabela 1).

\section{Tabela 1}

Avaliação das variáveis do ambiente alimentar do território com serviço de promoção da saúde. Belo Horizonte, Minas Gerais, Brasil, 2013.

\begin{tabular}{|c|c|c|}
\hline \multirow[t]{2}{*}{ Variáveis } & \multicolumn{2}{|c|}{ Estabelecimentos comerciais } \\
\hline & $\mathbf{n}$ & Valores \\
\hline Seção de frutas e hortaliças próxima à entrada principal & 262 & 78,0 \\
\hline \multicolumn{3}{|l|}{ Disponibilidade (\%) } \\
\hline Frutas (porções) & - & - \\
\hline Nenhuma & 4 & 1,2 \\
\hline $1-7$ & 122 & 36,3 \\
\hline $8-10$ & 210 & 62,5 \\
\hline Hortaliças (porções) & - & - \\
\hline Nenhuma & 7 & 2,1 \\
\hline $1-7$ & 174 & 51,8 \\
\hline $8-10$ & 155 & 46,1 \\
\hline \multicolumn{3}{|l|}{ Variedade (\%) } \\
\hline Frutas (porções) & - & - \\
\hline Nenhuma & 4 & 1,2 \\
\hline $1-14$ & 142 & 42,3 \\
\hline$\geq 15$ & 190 & 56,5 \\
\hline Hortaliças (porções) & - & - \\
\hline Nenhuma & 7 & 2,1 \\
\hline $1-14$ & 260 & 77,4 \\
\hline$\geq 15$ & 69 & 20,5 \\
\hline Disponibilidade de alimentos ultraprocessados (\%) & - & - \\
\hline Refrigerantes & 167 & 49,7 \\
\hline Variedade de marcas e sabores * & - & $15,0(9,0-24,5)$ \\
\hline Sucos adoçados e em pó & 173 & 51,5 \\
\hline Variedade de marcas * & - & $7,0(3,0-13,0)$ \\
\hline Biscoitos de chocolate recheados & 204 & 60,7 \\
\hline Variedade de marcas * & - & $6,0(3,2-8,0)$ \\
\hline Salgadinhos industrializados & 204 & 60,7 \\
\hline Variedade de marcas * & - & $2,0(2,0-4,0)$ \\
\hline \multicolumn{3}{|l|}{ Propaganda (\%) } \\
\hline Frutas e hortaliças & 74 & 22,0 \\
\hline Alimentos ultraprocessados & 256 & 76,2 \\
\hline \multicolumn{3}{|l|}{ Condições higiênico-sanitárias dos estabelecimentos (\%) } \\
\hline Aprovada & 34 & 11,3 \\
\hline Aprovada com restrições & 168 & 56,3 \\
\hline Reprovada & 96 & 32,4 \\
\hline
\end{tabular}

* Mediana e intervalo interquartil $\left(P_{25}-P_{75}\right)$. 
Os estabelecimentos apresentaram amplo horário de funcionamento, permanecendo em média abertos para o público por 12,4 $\pm 1,2$ horas durante a semana, 12,2 $\pm 1,4$ horas no sábado e 5,9 $\pm 2,1$ horas no domingo. Em relação à adequação higiênico-sanitária, 32,4\% dos estabelecimentos auditados foram reprovados e 56,3\% aprovados com restrições (Tabela 1). Os sacolões (38\%) e os mercados locais (34\%) foram os tipos de estabelecimentos comerciais que apresentaram as piores condições higiênicosanitárias (dados não apresentados).

$\mathrm{Na}$ avaliação da disponibilidade dos alimentos saudáveis, observou-se mediana do HFSI de 11 nos estabelecimentos investigados, variando de 5 a 16. Ao analisar esse índice de acordo com o tipo de estabelecimento, verificou-se que os sacolões e as feiras-livres apresentavam valores superiores $\left(\mathrm{HFSI}=13 ; \mathrm{P}_{25}-\mathrm{P}_{75}: 7-16\right)$ em comparação a mercados e supermercados de grande rede $(\mathrm{HFSI}=8$; $\left.\mathrm{P}_{25}-\mathrm{P}_{75}: 5-11 ; \mathrm{p}<0,001\right)$ e mercados locais (HFSI $\left.=7 ; \mathrm{P}_{25}-\mathrm{P}_{75}: 5-16 ; \mathrm{p}<0,001\right)$ (Figura 3).

\section{Discussão}

Este estudo revelou uma baixa validade das bases de dados secundárias. Houve a predominância de sacolões e feiras-livres, entretanto a disponibilidade de alimentos saudáveis foi insatisfatória, com presença importante de alimentos ultraprocessados e inadequadas condições higiênico-sanitárias dos estabelecimentos investigados.

O confrontamento entre bases de dados secundárias públicas com medidas objetivas do ambiente alimentar (auditoria) revelou baixa validade dos dados, em consonância com estudos conduzidos em diferentes localidades $3,21,22,23$. Neste trabalho, boa parte dos estabelecimentos registrados não existia, sendo que a cada dez estabelecimentos investigados somente quatro existiam, além de ser identificado um número expressivo de estabelecimentos não registrados nas bases.

Listas governamentais podem apresentar dados desatualizados por demora ou falha em remover estabelecimentos de alimentos que encerraram suas atividades 24 ou pela criação de novos estabelecimentos sem o registro junto aos órgãos fiscalizadores. Esses erros podem se concentrar em determinados tipos de estabelecimentos comerciais, como mercados de bairro 22, e em determinadas regiões, como aquelas com menor nível de urbanização ou maior nível de pobreza 23.

O uso pelos gestores de informações pouco válidas do ambiente alimentar pode prejudicar o investimento e o direcionamento de políticas de abastecimento de alimentos e esforços na melhoria do acesso à alimentação saudável 25. Ademais, a sua utilização em pesquisas pode comprometer a qualidade dos resultados, podendo igualmente repercutir em comprometimento do planejamento no território. Dessa forma, recomenda-se a validação das informações e a combinação de múltiplas fontes de dados, incluindo dados de agências reguladoras estaduais, municipais e de comércio 22, visando a garantir boa qualidade metodológica.

\section{Figura 3}

Distribuição do índice de acesso a alimentos em estabelecimentos de comercialização para consumo no domicílio (HFSI), de acordo com o tipo de estabelecimento comercial, no território com serviço de promoção da saúde. Belo Horizonte, Minas Gerais, Brasil, 2013.
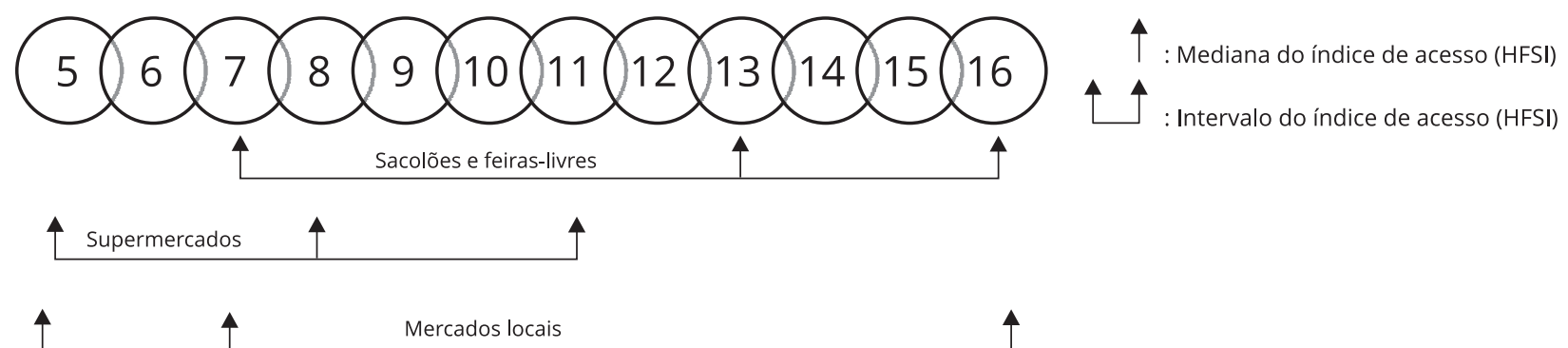
O uso de tecnologias como o Google Street View para a varredura das áreas analisadas constitui uma importante ferramenta para a validação dos dados. Entretanto, pondera-se que a validade e a obtenção dos dados com o uso da tecnologia pode ser influenciada pela data e horário da coleta da imagem e o não mapeamento de algumas regiões, sobretudo as rurais, mais pobres e pouco urbanizadas. Adicionalmente, pode desconsiderar ambientes fechados, nos quais a ferramenta não consegue realizar o mapeamento, como mercados e shoppings centers, subestimando o acesso 26. Apesar das limitações, o uso de ferramentas tecnológicas é relevante e crescente, sobretudo em grandes estudos por seu acesso fácil e gratuito.

A auditoria da área avaliada, como realizada neste trabalho, permite aferir de forma acurada a presença ou não do estabelecimento comercial e explorar o ambiente alimentar em todas as suas dimensões. Entretanto, em geral, ela é mais dispendiosa por requerer o deslocamento da equipe e sua capacitação, além da cobertura geográfica ser mais limitada 27, mas, ainda assim, é considerada método ouro na avaliação do ambiente alimentar 28.

Ao que se sabe este é o primeiro estudo no Brasil a investigar dados primários do ambiente alimentar da comunidade e do consumidor associados às condições higiênico-sanitárias dos estabelecimentos comerciais. Tais resultados podem contribuir para o delineamento de políticas públicas e para ampliar o conhecimento acerca do acesso à alimentação adequada e saudável no território.

A maior disponibilidade de alimentos saudáveis, verificada pelo HSFI, foi registrada nas feiras -livres e sacolões, em contraposição aos mercados e supermercados de grande rede. Um trabalho conduzido em áreas de baixa renda na cidade de São Paulo, também utilizando o HFSI, apresentou resultados semelhantes ${ }^{14}$. Num estudo conduzido em Baltimore, Estados Unidos, após ajuste por características sociodemográficas, a maior probabilidade de consumo de alimentos frescos também esteve associada à compra em sacolões ou feiras-livres 29. Entretanto, muitos estudos internacionais divergem desses resultados, mostrando os supermercados como marcadores de ambientes saudáveis e associados ao maior consumo de frutas e hortaliças, por apresentarem grande oferta e disponibilidade destes alimentos 21. Mas, pondera-se que em países desenvolvidos, onde a maior parte dos estudos de ambiente alimentar é desenvolvida, usualmente não apresentam estabelecimentos próprios para comercialização de frutas e hortaliças, como os sacolões no Brasil. Essa divergência possivelmente revela diferenças no delineamento do ambiente alimentar dos países, apontando a necessidade de ampliar a realização de estudos para cenários distintos.

Adicionalmente, observa-se uma grande contribuição dos supermercados para a compra de alimentos ultraprocessados no Brasil. Quase $2 / 3$ (60,4\%) da energia total adquirida com alimentos ultraprocessados provêm de supermercados 30. Tal resultado reforça a necessidade de elaboração de políticas públicas de abastecimento no Brasil, que favoreçam a implantação de estabelecimentos que comercializem predominantemente alimentos saudáveis, como sacolões e feiras-livres, e fortaleçam a agriculta familiar, promovendo maior acesso a uma maior rede saudável de distribuição 31,32.

Um estudo qualitativo conduzido com participantes do PAS de Belo Horizonte apontou como locais prioritários para a compra de alimentos saudáveis os mercados de bairro, seguidos dos sacolões 33 . Porém, neste trabalho, os mercados de bairro foram os estabelecimentos comerciais que apresentaram o pior índice de acesso a alimentos saudáveis. Apesar dos sacolões e feiras-livres ofertarem maior diversidade, qualidade e variedade de alimentos saudáveis 7 , os mercados de bairro parecem deter a preferência dos consumidores por fornecer atendimento diferenciado, tanto por permitir uma relação mais próxima entre comerciante e consumidor quanto por possibilitar compras a prazo 34 .

Em relação às condições higiênico-sanitárias, foi elevado o número de estabelecimentos comerciais com condições insatisfatórias, assim como verificado por Santos \& Bueno 35. A condição higiênicosanitária do comércio é considerada como um importante critério para a escolha do local de compra de frutas e hortaliças 33. Condições insatisfatórias podem favorecer a deterioração e a contaminação dos alimentos, sobretudo as frutas e hortaliças, levar a perdas e riscos à saúde dos consumidores 36 , além de fazer com que os consumidores percorram maiores distâncias para a compra de alimentos com boa qualidade 33. Acredita-se que a presença de estabelecimentos que vendem produtos frescos e que apresentam satisfatória qualidade higiênico-sanitária pode contribuir para o maior consumo de frutas e hortaliças 7 , sendo o investimento em fiscalização das condições sanitárias dos estabelecimentos e na promoção de ações educativas com os comerciantes estratégias importantes neste sentido. 
Como limitações deste estudo aponta-se não ter sido possível identificar todos os estabelecimentos de alguns dos territórios investigados $(\mathrm{n}=9)$ devido à localização geográfica limítrofe com municípios que não dispunham de bases de dados georreferenciadas. Ademais, este trabalho avaliou estabelecimentos comerciais de frutas e hortaliças, não sendo avaliados estabelecimentos que não tinham como atividade fim a venda destes itens (por exemplo, açougues, peixarias, confeitarias, entre outros). Esta foi uma escolha metodológica que pode ter limitado a compreensão do ambiente alimentar ao não incorporar todos os tipos de estabelecimentos presentes no território. Entretanto, acredita-se que boa parte dos estabelecimentos foi auditada, uma vez estes estabelecimentos quando comercializavam frutas e hortaliças também foram incluídos no estudo, além de terem sido avaliados outros 127 estabelecimentos para consumo no domicílio que não estavam contemplados na listagem fornecida pela Prefeitura.

Também não foram avaliados os estabelecimentos de comercialização de alimentos para consumo imediato. Entretanto, o estudo de Bezerra et al. (2013, apud Menezes et al. ${ }^{37}$ ) verificou que $40 \%$ da população brasileira consomem alimentos fora do domicílio, sendo este consumo menor em idosos $(18,9 \%)$, mulheres (39,5\%) e indivíduos com menor renda (36\%). Neste trabalho, a unidade de análise foi o território com PAS, de Belo Horizonte, no qual os usuários são, em sua maioria, mulheres (88,1\%), com idade média de $56,7 \pm 11,8$ anos ( $56,6 \%$ eram adultos mais velhos e $43,4 \%$ eram idosos), com baixo nível educacional $\left(7,2 \pm 4,1\right.$ anos) e renda mensal per capita $\left[\mathrm{R} \$ 678,0 ; \mathrm{P}_{25}-\mathrm{P}_{75}: 423,8-1000,0\right]$ 37. Dessa forma, acreditamos que trabalhamos em um contexto espacial marcado pela vulnerabilidade, onde, em geral, a maioria dos frequentadores possivelmente realizava suas refeições no próprio domicílio.

Outra limitação refere-se ao uso de buffers na definição da área investigada, determinando fronteiras arbitrárias. No entanto, utilizou-se valor de raio semelhante ao usado na literatura 12,13,34, além de ser uma distância razoavelmente grande que possibilita estabelecer distintos buffers de interesse e identificar outros tipos de deslocamentos e rotas.

Este estudo, ao investigar o ambiente alimentar de território com serviço de promoção da saúde do Sistema Único de Saúde (SUS), traz como potencial o alinhamento com políticas públicas de saúde. Dessa forma, pode contribuir para mapear as necessidades de implantação e reforço das ações de vigilância alimentar e nutricional (objetivando assegurar a qualidade nutricional dos alimentos disponíveis para aquisição/consumo e a promoção de práticas alimentares saudáveis) e de vigilância sanitária (visando a melhorar as condições higiênico-sanitárias dos estabelecimentos), bem como de políticas públicas de abastecimento de alimentos que favoreçam a construção de ambientes alimentares saudáveis 7 . Assim, sugere-se que o PAS se integre a políticas de abastecimento e comercialização de alimentos, articulando intersetorialmente com diferentes equipamentos, com vistas a propiciar escolhas alimentares adequadas e a saúde da população.

\section{Conclusão}

A baixa validade das bases de dados secundárias reforça a necessidade de realização de auditoria dos estabelecimentos comerciais visando à obtenção de resultados fidedignos, além da necessidade de ações de fiscalização mais eficientes.

No território foi predominante a presença de sacolões e feiras-livres, seguidos dos mercados e supermercados de grande rede. Apesar disso, foi insatisfatória a disponibilidade de alimentos saudáveis, com presença importante de alimentos ultraprocessados e inadequadas condições higiênicosanitárias dos estabelecimentos comerciais.

Reforça-se que esta investigação em conjunto do ambiente alimentar da comunidade e do consumidor associada às condições higiênico-sanitárias dos estabelecimentos comerciais contempla aspectos essenciais para o delineamento de políticas públicas de saúde, de alimentação e nutrição, que contribuam para assegurar o direito humano à alimentação adequada e saudável e à construção de ambientes alimentares saudáveis. 


\section{Colaboradores}

B. V. L. Costa e A. C. S. Lopes participaram da concepção do estudo, captação de recurso, coleta e análise dos dados, redação e revisão do manuscrito. P. P. Freitas, M. C. Menezes e L. M. F. Guimarães participaram da coleta e análise dos dados, redação e revisão do manuscrito. L. F. Ferreira e M. S. C. Alves participaram da coleta e análise dos dados. Todos os autores revisaram e aprovaram a versão final para publicação.

\section{Agradecimentos}

À Gerência de Cadastros Tributários da Secretaria Municipal Adjunta de Arrecadação de Belo Horizonte e à Gerência de Atenção Primária em Saúde da Secretaria Municipal da Saúde de Belo Horizonte. Este trabalho recebeu apoio financeiro do Conselho Nacional de Desenvolvimento Científico e Tecnológico (476686/2013-0) e da Fundação de Amparo à Pesquisa de Minas Gerais (PPM-0025415; APQ-03376-12).

\section{Referências}

1. Glanz K, Sallis JF, Saelens BE, Frank LD Healthy nutrition environments: concepts and measures. Am J Health Promot 2005; 19:330-3.

2. Kumanyika S. INFORMAS (International Network for Food and Obesity/non-communicable diseases Research, Monitoring and Action Support): summary and future directions. Obes Rev 2013; 14 Suppl 1:157-64.

3. Fleischhacker SE, Evenson KR, Sharkey J, Pitts SBJ, Rodriguez DA. Validity of secondary retail food outlet data a systematic review. J Prev Med 2013; 45:462-73.

4. Lucan SC. Concerning limitations of foodenvironment research: a narrative review and commentary framed around obesity and dietrelated diseases in youth. J Acad Nutr Diet 2015; 115:205-12.

5. Williams J, Scarborough P, Matthews A, Cowburn G, Foster C, Roberts N, et al. A systematic review of the influence of the retail food environment around schools on obesity-related outcomes. Obes Rev 2014; 15:359-74.

6. Farley TA, Baker ET, Futrell L, Rice JC. Ferreira $\mathrm{ABH}$. The ubiquity of energydense snack foods: a national multicity study. Am J Public Health 2010; 100:306-11.

7. Costa BVL, Oliveira CDL, Lopes ACS. Food environment of fruits and vegetables in the territory of the Health Academy Program. Cad Saúde Pública 2015; 31 Suppl:S159-69.

8. Skaba DA, Carvalho MS, Barcellos C, Martins PC, Terron SL. Geoprocessamento dos dados da saúde: o tratamento dos endereços. Cad Saúde Pública 2004; 20:1753-6.

9. Ministério da Saúde. Portaria no 2.681, de 7 de novembro de 2013. Redefine o Programa Academia da Saúde no âmbito do Sistema Único de Saúde (SUS). Diário Oficial da União 2013; 8 nov.

10. Costa BVL, Mendonça RD, Santos LC, Lopes ACS. Academia da Cidade: um serviço de promoção da saúde na rede assistencial do Sistema Único de Saúde. Ciênc Saúde Coletiva 2013; 18:95-102.

11. Furey S, Strugnell C, Mcllveen H. An investigation of the potential existence of "food deserts" in rural and urban areas of Northern Ireland. Agric Human Values 2001;18: 447-57.

12. Laska MN, Hearst MO, Forsyth A, Pasch KE, Lytle L. Neighbourhood food environments: are they associated with adolescent dietary intake, food purchases and weight status? Public Health Nutr 2010; 13:1757-63.

13. Hattori A, An R, Sturm R. Neighborhood food outlets, diet, and obesity among California adults, 2007 and 2009. Prev Chronic Dis 2013; 10:E35.

14. Duran AC, Roux AVD, Latorre M do RDO, Jaime PC. Neighborhood socioeconomic characteristics and differences in the availability of healthy food stores and restaurants in São Paulo, Brazil. Health Place 2013; 23:39-47. 
15. Duran AC, Lock K, Latorre MDRO, Jaime PC. Evaluating the use of in-store measures in retail food stores and restaurants in Brazil. Rev Saúde Pública 2015; 49:80.

16. Instituto Brasileiro de Geografia e Estatística. Pesquisa de Orçamentos Familiares 2008-2009. Antropometria e estado nutricional de crianças, adolescentes e adultos no Brasil. Rio de Janeiro: Instituto Brasileiro de Geografia e Estatística; 2010.

17. Cannuscio CC, Hillier A, Karpyn A, Glanz K. The social dynamics of healthy food shopping and store choice in an urban environment. Soc Sci Med 2014; 122:13-20.

18. Dover RVH, Lambert EV. "Choice Set” for health behavior in choiceconstrained settings to frame research and inform policy: examples of food consumption, obesity and food security. Int J Equity Health 2016; 15:48

19. Agência Nacional de Vigilância Sanitária. Resolução RDC no 275, de 21 de outubro de 2002. Dispõe sobre Regulamento Técnico de Procedimentos Operacionais Padronizados aplicados aos Estabelecimentos Produtores/Industrializadores de Alimentos e a Lista de Verificação das Boas Práticas de Fabricação em Estabelecimentos Produtores/Industrializadores de Alimentos. Diário Oficial da União 2002; 23 out.

20. Landis JR, Koch GG. The measurement of observer agreement for categorical data. Biometrics $1977 ; 33: 159-74$.

21. Caspi CE, Friebur R. Modified ground-truthing: an accurate and cost-effective food environment validation method for town and rural areas. Int J Behav Nutr Phys Act 2016; 13:37.

22. Liese AD, Colabianchi N, Lamichhane AP, Barnes TL, Hibbert JD, Porter DE, et al. Validation of 3 food outlet databases: completeness and geospatial accuracy in rural and urban food environments. Am J Epidemiol 2010; 172:1324-33.

23. Powell LM, Han E, Zenk SN, Khan T, Quinn $\mathrm{CM}$, Gibbs KP, et al. Field validation of secondary commercial data sources on the retail food outlet environment in the U.S. Health Place 2011; 17:1122-31.

24. Rossen LM, Pollack MK, Curriero FC. Verification of retail food outlet location data from a local health department using ground-truthing and remote-sensing technology: assessing differences by neighborhood characteristics. Health Place 2012 18:956-62.

25. Departamento de Análise de Situação de Saúde, Secretaria de Vigilância da Saúde, Ministério da Saúde. Política Nacional de Promoção da Saúde. Brasília: Ministério da Saúde; 2006. (Série B, Textos Básicos de Saúde).
26. Wilkins EW, Morris MA, Radley D, Griffiths C. Using Geographic Information Systems to measure retail food environments: discussion of methodological considerations and a proposed reporting checklist (Geo-FERN). Health Place 2017; 44:110-7.

27. Bader MD, Ailshire JA, Morenoff JD, House JS. Measurement of the local food environment: a comparison of existing data sources. Am J Epidemiol 2010; 171:609-17.

28. Partington SN, Menzies JT, Colburn AT, Saelens EB, Glanz K. Reduced-item food audits based on the nutrition environment measures surveys. Am J Prev Med 2015; 49:e23-33.

29. Machado P, Claro RM, Martins AP, Costa J, Levy-Costa R. Is food store type associated with the consumption of ultra-processed food and drink products in Brazil? Public Health Nutr 2018; 21:201-9.

30. Jilcott Pitts SB, Acheson MLM, Ward RK, Wu Q, McGuirt JT, Bullock SL, et al. Disparities in healthy food zoning, farmers' market availability, and fruit and vegetable consumption among North Carolina residents. Arch Public Health 2015; 73:35.

31. Lopes ACS, Menezes MC, Araújo ML. Food environment and access to fruits and vegetables: "a metropolis into perspective". Saúde Soc 2017; 26:764-73.

32. Vedovato GM, Surkan PJ, Jones-Smith J, Steeves EA, Han E, Trude AC, et al. Food insecurity, overweight and obesity among low-income African-American families in Baltimore City: associations with food-related perceptions. Public Health Nutr 2016; 19:1405-16.

33. Figueira TR, Lopes ACS, Modena CM. Avaliação do consumo de frutas e hortaliças entre famílias de usuários do Programa Academia da Saúde (PAS). Rev Bras Promoç Saúde 2014; 27:518-26.

34. Charreire H, Casey R, Salze P, Simon C, Chaix B, Banos A, et al. Measuring the food environment using geographical information systems: a methodological review. Public Health Nutr 2010; 13:1773-85.

35. Santos JT, Bueno M. Condições higiênico-sanitárias em setores de produtos perecíveis em supermercados no Vale do Paraíba. Rev Biociênc 2012; 18 (1 e 2 Esp):5-12.

36. Vilela NJ, Lana MM, Nascimento EF, Makishima N. O peso da perda de alimentos para a sociedade: o caso das hortaliças. Horticultura Brasileira 2003; 21:141-3.

37. Menezes MC, Costa BVL, Oliveira CDL, Lopes ACS. Local food environment and fruit and vegetable consumption: an ecological study. Prev Med Rep 2017; 5:13-20. 


\section{Abstract}

The study aimed to verify the validity of secondary data in the investigation of the food environment and to analyze the characteristics of the community environment and consumers in territories covered by a health promotion service. This was an ecological study in 18 units of the Health Academy Program in Belo Horizonte, Minas Gerais, Brazil, selected by simple cluster sampling. Validation of the establishments marketing fruits and vegetables, obtained from public databases, was done via telephone contact, Google Street View, and on-site audit. The following variables were investigated in the community food environment: type and location of the establishment; consumer's environment: availability, variety, price, and advertising of fruits and vegetables; availability and variety of ultra-processed foods; and hygienic and sanitary conditions. The access to healthy foods index was used to measure access to these foods. The on-site audit revealed weak concordance (45.7\%) with the secondary databases. Of the 298 establishments, the majority were bulk grocery stores and open-air markets (61.3\%), which showed the highest availability of healthy foods, but also marketed large amounts of ultra-processed foods (60.7\%). Onethird of the establishments showed substandard hygienic and sanitary conditions. The secondary databases showed low validity, emphasizing the need to audit the establishments. The establishments also showed a striking presence of ultraprocessed foods and poor hygiene and sanitation.

Commerce; Food; Urban Health; Validation Studies

\section{Resumen}

El objetivo fue verificar la validez de los datos secundarios en la investigación sobre el ambiente alimentario, así como analizar las características del ambiente de la comunidad y del consumidor, en territorios con servicios de promoción de la salud. Estudio ecológico, desarrollado en 18 unidades del Programa Academia de la Salud de Belo Horizonte, Minas Gerais, Brasil, seleccionadas por muestreo de conglomerado simple. La validación de los establecimientos que comercializan frutas y hortalizas, se obtuvo valiéndose de bases de datos públicas, se realizó por contacto telefónico, además del uso de la herramienta Google Street View y auditorías. Las variables del ambiente alimentario de la comunidad investigadas fueron: tipo de establecimiento y localización; ambiente del consumidor: disponibilidad, variedad, precio y propaganda de frutas y hortalizas, disponibilidad y variedad de alimentos ultraprocesados, además de aspectos higiénico-sanitarios. Para medir el acceso a alimentos saludables, se utilizó el índice de acceso a estos alimentos. La auditoría reveló una concordancia débil $(45,7 \%)$ de las bases secundarias de datos. De los 298 establecimientos auditados, la mayoría era tiendas de comestibles y mercados al aire libre (61,3\%), que presentaban una mayor disponibilidad de alimentos saludables, pero también comercializaban de forma expresiva alimentos ultraprocesados (60,7\%). En cuanto a las condiciones sanitarias, $1 / 3$ de los establecimientos suspendió. La validez de las bases secundarias fue baja, reforzando la necesidad de realizar auditorías en los establecimientos. Además, los establecimientos investigados presentaron una presencia expresiva de alimentos ultraprocesados e inadecuadas condiciones higiénico-sanitarias.

Comercio; Alimentos; Salud Urbana; Estudios de Validación
Recebido em 02/Out/2017

Versão final reapresentada em 18/Fev/2018

Aprovado em 18/Abr/2018 